\title{
MIRROR SYMMETRY AND LOCALIZATIONS
}

\author{
CHIU-CHU MELISSA LIU, CHIEN-HAO LIU, KEFENG LIU, AND SHING-TUNG YAU
}

\begin{abstract}
We describe the applications of localization methods, in particular the functorial localization formula, in the proofs of several conjectures from string theory. Functorial localization formula pushes the computations on complicated moduli spaces to simple moduli spaces. It is a key technique in the proof of the general mirror formula, the proof of the Hori-Vafa formula for explicit expressions of basic hypergeometric series of homogeneous manifolds, and the proof of the Mariño-Vafa formula for Hodge integrals. The proposal of Strominger-Yau-Zaslow of mirror symmetry will also be discussed.
\end{abstract}

\section{INTRODUCTION}

The main purpose of this lecture is to explain the applications of a variation of the localization formula of Atiyah-Bott in solving various conjectures from string theory. This variation we call the Functorial Localization Formula. We will also discuss the role of the SYZ proposal in mirror symmetry.

We start with a review of the Atiyah-Bott localization formula. Recall that the definition of equivariant cohomology group for a manifold $X$ with a torus $T$ action:

$$
H_{T}^{*}(X)=H^{*}\left(X \times_{T} E T\right)
$$

where $E T$ is the universal bundle of $T$.

Example. We know $E S^{1}=S^{\infty}$. If $S^{1}$ acts on $\mathbf{P}^{n}$ by

$$
\lambda \cdot\left[Z_{0}, \ldots, Z_{n}\right]=\left[\lambda^{w_{0}} Z_{0}, \ldots, \lambda^{w_{n}} Z_{n}\right],
$$

then

$$
H_{S^{1}}\left(\mathbf{P}^{n} ; \mathbb{Q}\right) \cong \mathbb{Q}[H, \alpha] /\left\langle\left(H-w_{0} \alpha\right) \cdots\left(H-w_{n} \alpha\right)\right\rangle
$$

where $\alpha$ is the generator of $H^{*}\left(B S^{1}, \mathbb{Q}\right)$.

Atiyah-Bott Localization Formula. For $\omega \in H_{T}^{*}(X)$ an equivariant cohomology class, we have

$$
\omega=\sum_{E} i_{E *}\left(\frac{i_{E}^{*} \omega}{e_{T}(E / X)}\right) .
$$

where $E$ runs over all connected components of $T$ fixed points set.

This formula is very effective in the computations of integrals on manifolds with torus $T$ symmetry. The idea of localization is fundamental in many subjects of geometry. In fact Atiyah and Witten proposed to formally apply this localization formula to loop spaces and the natural $S^{1}$-action, from which one gets the AtiyahSinger index formula. In fact the Chern characters can be interpreted as equivariant forms on loop space, and the $\hat{A}$-class is the inverse of the equivariant Euler class of the normal bundle of $X$ in its loop space $L X$ :

$$
e_{T}(X / L X)^{-1} \sim \hat{A}(X),
$$


which follows from the normalized infinite product formula

$$
\left(\prod_{n \neq 0}(x+n)\right)^{-1} \sim \frac{x}{\sin x} .
$$

K. Liu observed in [Liu] that the normalized product

$$
\prod_{m, n}(x+m+n \tau)=2 q^{\frac{1}{8}} \sin (\pi x) \cdot \prod_{j=1}^{\infty}\left(1-q^{j}\right)\left(1-e^{2 \pi i x} q^{j}\right)\left(1-e^{-2 \pi i x} q^{j}\right),
$$

where $q=e^{2 \pi i \tau}$, also has deep geometric meaning. This formula is the Eisenstein formula. It can be viewed as a double loop space analogue of the Atiyah-Witten observation. This formula gives the basic Jacobi $\theta$-function. As observed by K. Liu, formally this gives the $\hat{A}$-class of the loop space, and the Witten genus which is defined to be the index of the Dirac operator on the loop space:

$$
e_{T}(X / L L X) \sim \hat{W}(X),
$$

where $L L X$ is the double loop space, the space of maps from $S^{1} \times S^{1}$ into $X . \hat{W}(X)$ is the Witten class. See [Liu] for more detail.

The variation of the localization formula we will use in various situations is the following

Functorial Localization Formula. Let $X$ and $Y$ be two manifolds with torus action. Let $f: X \rightarrow Y$ be an equivariant map. Given $F \subset Y$ a fixed component, let $E \subset f^{-1}(F)$ be those fixed components inside $f^{-1}(F)$. Let $f_{0}=\left.f\right|_{E}$, then for $\omega \in H_{T}^{*}(X)$ an equivariant cohomology class, we have the following identity on $F$ :

$$
f_{0 *}\left[\frac{i_{E}^{*} \omega}{e_{T}(E / X)}\right]=\frac{i_{F}^{*}\left(f_{*} \omega\right)}{e_{T}(F / Y)} .
$$

This formula will be applied to various settings to prove the conjectures from physics. It first appeared in [L-L-Y1, I]. It is used to push computations on complicated moduli spaces to simpler moduli spaces. A $K$-theory version of the functorial localization formula also holds [L-L-Y1, II], interesting applications are expected.

Remark. Consider the diagram:

$$
\begin{array}{ccc}
H_{T}^{*}(X) & \stackrel{f_{*}}{\longrightarrow} & H_{T}^{*}(Y) \\
\downarrow i_{E}{ }^{*} & & \downarrow i_{F}{ }^{*} \\
H_{T}^{*}(E) & \stackrel{f_{0_{*}}}{\longrightarrow} & H_{T}^{*}(F) .
\end{array}
$$

The functorial localization formula is like Riemann-Roch with the inverted equivariant Euler classes of the normal bundle as "weights", in a way similar to the Todd class for the Riemann-Roch formula. In fact if we formally apply this formula to the map between the loop spaces of $X$ and $Y$, equivariant with respect to the rotation of the circle, we do formally get the differentiable Riemann-Roch formula. We believe this can be done rigorously by following Bismut's proof of the index formula which made rigorous of the above argument of Atiyah-Witten.

This formula will be used in the following setups: 
(1). The proof of the mirror formulas and its generalizations which we call the mirror principle. The mirror principle implies all of the conjectural formulas for toric manifolds and their Calabi-Yau submanifolds from string theory. In this case we apply the functorial localization formula to the map from the nonlinear moduli space to the linearized moduli space. This transfers the computations of integrals on complicated moduli space of stable maps to computations on rather simple spaces like projective spaces. From this the proof of the mirror formula and its generalizations become conceptually clean and simple.

In fact the functorial localization formula was first found and used in Lian-LiuYau's proof of the mirror conjecture.

(2). The proof of the Hori-Vafa conjecture and its generalizations for Grassmannian and flag manifolds. This conjecture predicts an explicit formula for the basic hypergeometric series of a homogeneous manifold in terms of the basic series of a simpler manifold such as the product of projective spaces. In this case we use the functorial localization formula twice to transfer the computations on the complicated moduli spaces of stable curves to the computations on quot-schemes. The first is a map from moduli space of stable maps to product of projective spaces, and another one is a map from the quot-scheme into the same product of projective spaces. A key observation we had is that these two maps have the same image.

This approach was first sketched in [L-L-Y1, III], the details for Grassmannians were carried out in [L-L-L-Y] and [B-CF-K]. The most general case of flag manifolds was carried out in [ChL-L-Y2].

(3). The proof of a remarkable conjecture of Mariño-Vafa on Hodge integrals by C.-C. M. Liu, K. Liu and Z. Zhou [L-L-Z1]. This conjecture gives a closed formula for the generating series of a class of triple Hodge integrals for all genera and any number of marked points in terms of the Chern-Simons knot invariant of the unknot. This formula was conjectured by M. Mariño and C. Vafa in $[\mathrm{M}-\mathrm{V}]$ based on the duality between large $N$ Chern-Simons theory and string theory. Many Hodge integral identities, including the ELSV formula for Hurwitz numbers [ELSV] and the $\lambda_{g}$ conjecture [Ge-P, Fa-P2], can be obtained by taking various limits of the Mariño-Vafa formula [L-L-Z2]. The Mariño-Vafa formula was proved by applying the functorial localization formula to the branch morphism from the moduli space of relative stable maps to a projective space.

\section{Mirror Principle}

There have been many discussion of mirror principle in the literature. Here we only give a brief account of the main ideas of the setup and proof of the mirror principle. We will use two most interesting examples to illustrate the algorithm.

The goal of mirror principle is to compute the characteristic numbers on moduli spaces of stable maps in terms of certain hypergeometric type series. This was motivated by mirror symmetry in string theory. The most interesting case is the counting of the numbers of curves which corresponds to the computations of Euler numbers. More generally we would like to compute the characteristic numbers and classes induced from the general Hirzebruch multiplicative classes such as the total Chern classes. The computations of integrals on moduli spaces of those classes pulled back through evaluation maps at the marked points and the general GromovWitten invariants can also be considered as part of mirror principle. Our hope 
is to develop a "black-box" method which makes easy the computations of the characteristic numbers and the Gromov-Witten invariants.

The general set-up of mirror principle is as follows. Let $X$ be a projective manifold, $\mathcal{M}_{g, k}(d, X)$ be the moduli space of stable maps of genus $g$ and degree $d$ with $k$ marked points into $X$, modulo the obvious equivalence. The points in $\mathcal{M}_{g, k}(d, X)$ are triples $\left(f ; C ; x_{1}, \cdots, x_{k}\right)$ where $f: C \rightarrow X$ is a degree $d$ holomorphic map and $x_{1}, \cdots, x_{k}$ are $k$ distinct smooth points on the genus $g$ curve $C$. The homology class $f_{*}([C])=d \in H_{2}(X, \mathbb{Z})$ is identified as integral index $d=\left(d_{1}, \cdots, d_{n}\right)$ by choosing a basis of $H_{2}(X, \mathbb{Z})$, dual to the Kähler classes.

In general the moduli space may be very singular, and may even have different dimension for different components. To define integrals on such singular spaces, we need the virtual fundamental cycle of Li-Tian [L-T], and also Behrend-Fantechi $[\mathrm{B}-\mathrm{F}]$ which we denote by $\left[\mathcal{M}_{g, k}(d, X)\right]^{v}$. This is a homology class of the expected dimension

on $\mathcal{M}_{g, k}(d, X)$.

$$
2\left(c_{1}(T X)[d]+\left(\operatorname{dim}_{\mathbb{C}} X-3\right)(1-g)+k\right)
$$

Let us consider the case $k=0$ first. Note that the expected dimension of the virtual fundamental cycle is 0 if $X$ is a Calabi-Yau 3 -fold. This is the most interesting case for string theory.

The starting data of mirror principle are as follows. Let $V$ be a concavex bundle on $X$ which we defined as the direct sum of a positive and a negative bundle on $X$. Then $V$ induces a sequence of vector bundles $V_{d}^{g}$ on $\mathcal{M}_{g, 0}(d, X)$ whose fiber at $\left(f ; C ; x_{1}, \cdots, x_{k}\right)$ is given by $H^{0}\left(C, f^{*} V\right) \oplus H^{1}\left(C, f^{*} V\right)$. Let $b$ be a multiplicative characteristic class. So far for all applications in string theory, $b$ is the Euler class.

The problem of mirror principle is to compute

$$
K_{d}^{g}=\int_{\left[\mathcal{M}_{g, 0}(d, X)\right]^{v}} b\left(V_{d}^{g}\right) .
$$

More precisely we want to compute the generating series

$$
F(T, \lambda)=\sum_{d, g} K_{d}^{g} \lambda^{g} e^{d \cdot T}
$$

in terms of certain hypergeometric type series. Here $\lambda, T=\left(T_{1}, \cdots, T_{n}\right)$ are formal variables.

The most famous formula in the subject is the Candelas formula as conjectured by P. Candelas, X. de la Ossa, P. Green, and L. Parkes [CdGP]. This formula changed the history of the subject. More precisely, Candelas formula considers the genus 0 curves, that is, we want to compute the so-called $A$-model potential of a Calabi-Yau 3-fold $M$ given by

$$
\mathcal{F}_{0}(T)=\sum_{d \in H_{2}(M ; \mathbb{Z})} K_{d}^{0} e^{d \cdot T},
$$

where $T=\left(T_{1}, \ldots, T_{n}\right)$ are considered as the coordinates of the Kahler moduli of $M$, and $K_{d}^{0}$ is the genus zero, degree $d$ invariant of $M$ which gives the numbers of rational curves of all degree through the multiple cover formula [L-L-Y1]. The famous mirror conjecture asserts that there exists a mirror Calabi-Yau 3-fold $M^{\prime}$ with $B$-model potential $\mathcal{G}(T)$, which can be computed by period integrals, such that

$$
\mathcal{F}(T)=\mathcal{G}(t),
$$


where $t$ accounts for coordinates of complex moduli of $M^{\prime}$. The map $t \mapsto T$ is called the mirror map. In the toric case, the period integrals are explicit solutions to the GKZ-system, that is the Gelfand-Kapranov-Zelevinsky hypergeometric series. While the A-series are usually very difficult to compute, the B-series are very easy to get. This is the magic of the mirror formula. We will discuss the proof of the mirror principle which includes the proof of the mirror formula.

The key ingredients for the proof of the mirror principle consists of

(1) Linear and non-linear moduli spaces;

(2) Euler data and hypergeometric (HG) Euler data.

More precisely, the non-linear moduli is the moduli space $M_{d}^{g}(X)$ which is the stable map moduli of degree $(1, d)$ and genus $g$ into $\mathbf{P}^{1} \times X$. A point in $M_{d}^{g}(X)$ consists of a pair $\left.(f, C): f: C \rightarrow \mathbf{P}^{1} \times X\right\}$ with $C$ a genus $g$ (nodal) curve, modulo obvious equivalence. The linearized moduli $W_{d}$ for toric $X$ were first introduced by Witten and used by Aspinwall-Morrison to do approximating computations.

Example. Consider the projective space $\mathbf{P}^{n}$ with homogeneous coordinate $\left[z_{0}, \cdots, z_{n}\right]$. Then the linearized moduli $W_{d}$ is defined as projective space with homogeneous coordinates $\left[f_{0}\left(w_{0}, w_{1}\right), \cdots, f_{n}\left(w_{0}, w_{1}\right)\right]$ where $f_{j}\left(w_{0}, w_{1}\right)$ 's are homogeneous polynomials of degree $d$.

This is the simplest compactification of the moduli spaces of degree $d$ maps from $\mathbf{P}^{1}$ into $\mathbf{P}^{n}$. The following lemma is important. See [L-L-Y1, IV] for its proof. The $g=0$ case was given in $[\mathrm{Gi}]$ and in $[\mathrm{L}-\mathrm{L}-\mathrm{Y} 1, \mathrm{I}]$.

Lemma 1. There exists an explicit equivariant collapsing map

$$
\varphi: M_{d}^{g}\left(\mathbf{P}^{n}\right) \longrightarrow W_{d}
$$

For general projective manifold $X$, the nonlinear moduli $M_{d}^{g}(X)$ can be embedded into $M_{d}^{g}\left(\mathbf{P}^{n}\right)$. The nonlinear moduli $M_{d}^{g}(X)$ is very "singular" and complicated, but the linear moduli $W_{d}$ is smooth and simple. The embedding induces a map of $M_{d}^{g}(X)$ to $W_{d}$. Functorial localization formula pushed the computations onto $W_{d}$. Usually mathematical computations should be done on the moduli of stable maps, while physicists tried to use the linearized moduli to approximate the computations. So functorial localization formula connects the computations of mathematicians and physicists. In some sense the mirror symmetry formula is more or less the comparison of computations on nonlinear and linearized moduli.

Mirror principle has been proved to hold for balloon manifolds. A projective manifold $X$ is called balloon manifold if it admits a torus action with isolated fixed points, and if the following conditions hold. Let

$$
H=\left(H_{1}, \cdots, H_{k}\right)
$$

be a basis of equivariant Kahler classes such that

(1) the restrictions $H(p) \neq H(q)$ for any two fixed points $p \neq q$;

(2) the tangent bundle $T_{p} X$ has linearly independent weights for any fixed point $p$.

This notion was introduced by Goresky-Kottwitz-MacPherson.

Theorem 2. Mirror principle holds for balloon manifolds and for any concavex bundles. 


\section{Remarks.}

1. All toric manifolds are balloon manifolds. For $g=0$ we can identify the hypergeometric series explicitly. Higher genus cases need more work to identify such series.

2. For toric manifolds and $g=0$, mirror principle implies all of the mirror conjectural formulas from string theory.

3. For Grassmannian manifolds, the explicit mirror formula is given by the Hori-Vafa formula to be discussed in Section 3.

4. The case of direct sum of positive line bundles on $\mathbf{P}^{n}$, including the Candelas formula, has two independent approaches by Givental, and by LianLiu-Yau.

Now we briefly discuss the proof of the mirror principle. The main idea is to apply the functorial localization formula to $\varphi$, the collapsing map and the pull-back class $\omega=\pi^{*} b\left(V_{d}^{g}\right)$, where $\pi: \mathcal{M}_{d}^{g}(X) \rightarrow M_{g, 0}(d, X)$ is the natural projection.

Such classes satisfy certain induction property. To be precise we introduce the notion of Euler Data, which naturally appears on the right hand side of the functorial localization formula, $Q_{d}=\varphi_{!}\left(\pi^{*} b\left(V_{d}^{g}\right)\right)$ which is a sequence of polynomials in equivariant cohomology rings of the linearized moduli spaces with simple quadratic relations. We also considered their restrictions to $X$.

From functorial localization formula we prove that, by knowing the Euler data $Q_{d}$ we can determine the $K_{d}^{g}$. On the other hand, there is another much simpler Euler data, the $H G$ Euler data $P_{d}$, which coincides with $Q_{d}$ on the "generic" part of the nonlinear moduli. We prove that the quadratic relations and the coincidence on generic part determine the Euler data uniquely up to certain degree. We also know that $Q_{d}$ always have the right degree for $g=0$. We then use mirror transformation to reduce the degrees of the HG Euler data $P_{d}$. From these we deduced the mirror principle.

\section{Remarks.}

1. Both the denominator and the numerator in the HG series, the generating series of the HG Euler data, are equivariant Euler classes. Especially the denominator is exactly from the localization formula. This is easily seen from the functorial localization formula.

2. The quadratic relation of Euler data, which naturally comes from gluing and functorial localization on the A-model side, is closely related to special geometry, and is similar to the Bershadsky-Cecotti-Ooguri-Vafa's holomorphic anomaly equation on the B-model side. Such relation can determine the polynomial Euler data up to certain degree.

It is an interesting task to use special geometry to understand the mirror principle computations, especially the mirror transformation as a coordinate change.

3. The Mariño-Vafa formula to be discussed in Section 4 is needed to determine the hypergeometric Euler data for higher genus computations in mirror principle. The Mariño-Vafa formula comes from the duality between ChernSimons theory and Gromov-Witten theory. This duality and the matrix model for Chern-Simons theory indicate that mirror principle may have matrix model description.

Let us use two examples to illustrate the algorithm of mirror principle. 
Example. Consider the Calabi-Yau quintic in $\mathbf{P}^{4}$. In this case

$$
P_{d}=\prod_{m=0}^{5 d}(5 \kappa-m \alpha)
$$

with $\alpha$ can be considered as the weight of the $S^{1}$ action on $\mathbf{P}^{1}$, and $\kappa$ denotes the generator of the equivariant cohomology ring of $W_{d}$.

The starting data of the mirror principle in this case is $V=\mathcal{O}(5)$ on $X=\mathbf{P}^{4}$. The hypergeometric series, after taking $\alpha=-1$, is given by

$$
H G[B](t)=e^{H t} \sum_{d=0}^{\infty} \frac{\prod_{m=0}^{5 d}(5 H+m)}{\prod_{m=1}^{d}(H+m)^{5}} e^{d t}
$$

where $H$ is the hyperplane class on $\mathbf{P}^{4}$ and $t$ is a formal parameter.

We introduce the series

$$
\mathcal{F}(T)=\frac{5}{6} T^{3}+\sum_{d>0} K_{d}^{0} e^{d T} .
$$

The algorithm is as follows. Take the expansion in $H$ :

$$
H G[B](t)=H\left\{f_{0}(t)+f_{1}(t) H+f_{2}(t) H^{2}+f_{3}(t) H^{3}\right\},
$$

from which we have the famous Candelas Formula: With $T=f_{1} / f_{0}$,

$$
\mathcal{F}(T)=\frac{5}{2}\left(\frac{f_{1}}{f_{0}} \frac{f_{2}}{f_{0}}-\frac{f_{3}}{f_{0}}\right) .
$$

Example. Let $X$ be a toric manifold and $g=0$. Let $D_{1}, \ldots, D_{N}$ be the $T$-invariant divisors in $X$. The starting data consist of $V=\oplus_{i} L_{i}$ with $c_{1}\left(L_{i}\right) \geq 0$ and $c_{1}(X)=$ $c_{1}(V)$. Let us take $b(V)=e(V)$ the Euler class. We want to compute the A-series

$$
A(T)=\sum K_{d}^{0} e^{d \cdot T} .
$$

The HG Euler series which is the generating series of the HG Euler data can be easily written down as

$$
B(t)=e^{-H \cdot t} \sum_{d} \prod_{i} \prod_{k=0}^{\left\langle c_{1}\left(L_{i}\right), d\right\rangle}\left(c_{1}\left(L_{i}\right)-k\right) \frac{\prod_{\left\langle D_{a}, d\right\rangle<0} \prod_{k=0}^{-\left\langle D_{a}, d\right\rangle-1}\left(D_{a}+k\right)}{\prod_{\left\langle D_{a}, d\right\rangle \geq 0} \prod_{k=1}^{\left\langle D_{a}, d\right\rangle}\left(D_{a}-k\right)} e^{d \cdot t} .
$$

Then mirror principle implies that there are explicitly computable functions $f(t), g(t)$, which define the mirror map, such that

$$
\int_{X}\left(e^{f} B(t)-e^{-H \cdot T} e(V)\right)=2 A(T)-\sum T_{i} \frac{\partial A(T)}{\partial T_{i}}
$$

where $T=t+g(t)$. From this equation we can easily solve for $A(T)$.

In general we want to compute:

$$
K_{d, k}^{g}=\int_{\left[\mathcal{M}_{g, k}(d, X)\right]^{v}} \prod_{j=1}^{k} e v_{j}^{*} \omega_{j} \cdot b\left(V_{d}^{g}\right)
$$

where $\omega_{j} \in H^{*}(X)$ and $e v_{j}$ denotes the evaluation map at the $j$-th marked point. We form a generating series with $t, \lambda$ and $\nu$ formal variables,

$$
F(t, \lambda, \nu)=\sum_{d, g, k} K_{d, k}^{g} e^{d t} \lambda^{2 g} \nu^{k}
$$


The ultimate mirror principle we want to prove is to compute this series in terms of certain explicit HG series. It is easy to show that those classes in the integrand can still be combined to induce Euler data. Actually the Euler data really encode the geometric structure of the stable map moduli.

We only use one example to illustrate the higher genus mirror principle.

Example. Consider open toric Calabi-Yau manifold, say $\mathcal{O}(-3) \rightarrow \mathbf{P}^{2}$. Here $V=$ $\mathcal{O}(-3)$. Let

$$
Q_{d}=\sum_{g \geq 0} \varphi_{!}\left(\pi^{*} e_{T}\left(V_{d}^{g}\right)\right) \lambda^{2 g}
$$

Then it can be shown that the corresponding HG Euler data is given explicitly by

$$
P_{d} J(\kappa, \alpha, \lambda) J(\kappa-d \alpha,-\alpha, \lambda),
$$

where $P_{d}$ is exactly the genus 0 HG Euler data and $J$ is generating series of Hodge integrals with summation over all genera. $J$ may be considered as the degree 0 Euler data. In fact we may say that the computations of Euler data include computations of all Gromov-Witten invariants, and even more. Some closed formulas can be obtained. We have proved that the mirror principle holds in such general setting. The remaining task is to determine the explicit HG Euler data.

Finally we mention some recent works. First we have constructed refined linearized moduli space for higher genus, the $A$-twisted moduli stack $\mathcal{A M}_{g}(X)$ of genus $g$ curves associated to a smooth toric variety $X$, induced from the gauged linear sigma model studied by Witten.

This new moduli space is constructed as follows. A morphism from a curve of genus $g$ into $X$ corresponds to an equivalence class of triples $\left(L_{\rho}, u_{\rho}, c_{m}\right)_{\rho, m}$, where each $L_{\rho}$ is a line bundle pulled back from $X, u_{\rho}$ is a section of $L_{\rho}$ satisfying a nondegeneracy condition, and the collection $\left\{c_{m}\right\}_{m}$ gives conditions to compare the sections $u_{\rho}$ in different line bundles $L_{\rho}$, (cf.[Cox]). $\mathcal{A M}_{g}(X)$ is the moduli space of such data. It is an Artin stack, fibered over the moduli space of quasi-stable curves [ChL-L-Y1]. We hope to use this refined moduli to do computations for higher genus mirror principle.

On the other hand, motivated by recent progresses in open string theory, we are also trying to develop open mirror principle. Open string theory predicts formulas for the counting of holomorphic discs with boundary inside a Lagrangian submanifold, more generally of the counting of the numbers of open Riemann surfaces with boundary in Lagrangian submanifold. Linearized moduli space for such data is being constructed which gives a new compactification of such moduli spaces.

\section{Hori-VAfa Formula}

In $[\mathrm{H}-\mathrm{V}]$, Hori and Vafa generalize the world-sheet aspects of mirror symmetry to being the equivalence of $d=2, N=(2,2)$ supersymmetric field theories (i.e. without imposing the conformal invariance on the theory). This leads them to a much broader encompassing picture of mirror symmetry. See [HKKPTVVZ] for full explanations. Putting this in the frame work of abelian gauged linear sigma models (GLSM) [Wi1] enables them to link many $d=2$ field theories together. Generalization of this setting to nonabelian GLSM ([Wi1, Section 5.3]) leads them to the following conjecture, when the physical path integrals are interpreted appropriately mathematically: 
Conjecture 3 (Hori-Vafa $[\mathrm{H}-\mathrm{V}$, Appendix A]). The hypergeometric series for a given homogeneous space (e.g. a Grassmannian manifold) can be reproduced from the hypergeometric series of simpler homogeneous spaces (e.g. product of projective spaces). Similarly for the twisted hypergeometric series that are related to the submanifolds in homogeneous spaces.

In other words, different homogeneous spaces (or some simple quotients of them) can give rise to generalized mirror pairs.

Some progress towards this conjecture has been made for general flag manifolds by using hyper-quot schemes in [ChL-L-Y2]. The derivation of the formula for flag manifolds is rather complicated, involving many technical new ingredients like restrictive flag manifolds. A main object to be understood in the above conjecture is the fundamental hypergeometric series $H G[\mathbf{1}]^{X}(t)$ associated to the flag manifold $X$. Recall that in the computations of mirror principle, the existence of linearized moduli made easy the computations for toric manifolds.

An outline of how this series may be computed was given in [L-L-Y1, III] via an extended mirror principle diagram. To make clear the main ideas we will only focus on the case of Grassmannian manifolds in this article. The main problem for the computation is that there is no known good linearized moduli for Grassmannian or general flag manifolds. To overcome the difficulty we use the Grothendieck quot scheme to play the role of the linearized moduli. The method gives a complete proof of the Hori-Vafa formula in the Grassmannian case.

Let $e v: \mathcal{M}_{0,1}(d, X) \rightarrow X$ be the evaluation map on the moduli space of stable maps with one marked point, and $c$ the first Chern class of the tangent line at the marked point. The fundamental hypergeometric series for mirror formula is given by the push-forward:

$$
e v_{*}\left[\frac{1}{\alpha(\alpha-c)}\right] \in H^{*}(X)
$$

or more precisely the generating series

$$
H G[1]^{X}(t)=e^{-t H / \alpha} \sum_{d=0}^{\infty} e v_{*}\left[\frac{1}{\alpha(\alpha-c)}\right] e^{d t} .
$$

Assume the linearized moduli exists. Then functorial localization formula applied to the collapsing map: $\varphi: M_{d} \rightarrow N_{d}$, immediately gives the expression as the denominator of the hypergeometric series.

Example. $X=\mathbf{P}^{n}$, then we have $\varphi_{*}(1)=1$, functorial localization immediately gives us

$$
e v_{*}\left[\frac{1}{\alpha(\alpha-c)}\right]=\frac{1}{\prod_{m=1}^{d}(x-m \alpha)^{n+1}}
$$

where the denominators of both sides are equivariant Euler classes of normal bundles of the fixed points. Here $x$ denotes the hyperplane class.

For $X=\operatorname{Gr}(k, n)$ or general flag manifolds, no explicit linearized moduli is known. Hori-Vafa conjectured a formula for $H G[1]^{X}(t)$ by which we can compute this series in terms of those of projective spaces: 
Hori-Vafa Formula for Grassmannians. We have

$H G[1]^{\operatorname{Gr}(k, n)}(t)=\left.\frac{e^{(k-1) \pi \sqrt{-1} \sigma / \alpha}}{\prod_{i<j}\left(x_{i}-x_{j}\right)} \cdot \prod_{i<j}\left(\alpha \frac{\partial}{\partial x_{i}}-\alpha \frac{\partial}{\partial x_{j}}\right)\right|_{t_{i}=t+(k-1) \pi \sqrt{-1}} H G[1]^{\mathbf{P}}\left(t_{1}, \cdots, t_{k}\right)$

where $\mathbf{P}=\mathbf{P}^{n-1} \times \cdots \times \mathbf{P}^{n-1}$ is product of $k$ copies of the projective spaces, $\sigma$ is the generator of the divisor classes on $\operatorname{Gr}(k, n)$ and $x_{i}$ the hyperplane class of the $i$-th copy $\mathbf{P}^{n-1}$ :

$$
H G[1]^{\mathbf{P}}\left(t_{1}, \cdots, t_{k}\right)=\prod_{i=1}^{k} H G[1]^{\mathbf{P}^{\mathbf{n}-1}}\left(t_{i}\right) .
$$

Now we describe the ideas of the proof of the above formula. As mentioned above we use another smooth moduli space, the Grothendieck quot-scheme $Q_{d}$ to play the role of the linearized moduli, and apply the functorial localization formula. Here is the general set-up:

To start, note that the Plücker embedding $\tau: \operatorname{Gr}(k, n) \rightarrow \mathbf{P}^{N}$ induces an embedding of the nonlinear moduli $M_{d}$ of $\operatorname{Gr}(k, n)$ into that of $\mathbf{P}^{N}$. Composite of this map with the collapsing map gives us a map $\varphi: M_{d} \rightarrow W_{d}$ into the linearized moduli space $W_{d}$ of $\mathbf{P}^{N}$. On the other hand the Plücker embedding also induces a map $\psi: Q_{d} \rightarrow W_{d}$. We have the following three crucial lemmas proved in [L-L-L-Y].

Lemma 4. The above two maps have the same image in $W_{d}: \operatorname{Im} \psi=\operatorname{Im} \varphi$. And all the maps are equivariant with respect to the induced circle action from $\mathbf{P}^{1}$.

Just as in the mirror principle computations, our next step is to analyze the fixed points of the circle action induced from $\mathbf{P}^{1}$. In particular we need the distinguished fixed point set to get the equivariant Euler class of its normal bundle. The distinguished fixed point set in $M_{d}$ is $\mathcal{M}_{0,1}(d, \operatorname{Gr}(k, n))$ with equivariant Euler class of its normal bundle given by $\alpha(\alpha-c)$, and we know that $\varphi$ is restricted to $e v$.

Lemma 5. The distinguished fixed point set in $Q_{d}$ is a union: $\cup_{s} E_{0 s}$, where each $E_{0 s}$ is a fiber bundle over $\operatorname{Gr}(k, n)$ with fiber given by flag manifold.

It is a complicated work to determine the fixed point sets $E_{0 s}$ and the weights of the circle action on their normal bundles. The situation for flag manifold cases are much more involved. See [L-L-L-Y] and [ChL-L-Y2] for details.

Now let $p$ denote the projection from $E_{0 s}$ onto $\operatorname{Gr}(k, n)$. Functorial localization formula, applied to $\varphi$ and $\psi$, gives us the following

Lemma 6. We have the equality on $\operatorname{Gr}(k, N)$ :

$$
e v_{*}\left[\frac{1}{\alpha(\alpha-c)}\right]=\sum_{s} p_{*}\left[\frac{1}{e_{T}\left(E_{0 s} / Q_{d}\right)}\right]
$$

where $e_{T}\left(E_{0 s} / Q_{d}\right)$ is the equivariant Euler class of the normal bundle of $E_{0 s}$ in $Q_{d}$.

Finally we compute $p_{*}\left[\frac{1}{e_{T}\left(E_{0 s} / Q_{d}\right)}\right]$. There are two different approaches, the first one is by direct computations in [L-L-L-Y], and another one is by using the wellknown Euler sequences for universal sheaves [B-CF-K]. The second method has the advantage of being more explicit. Note that

$$
e_{T}\left(\left.T Q\right|_{E_{0 s}}-T E_{0 s}\right)=e_{T}\left(\left.T Q\right|_{E_{0 s}}\right) / e_{T}\left(T E_{0 s}\right) .
$$


Both $e_{T}\left(\left.T Q\right|_{E_{0 s}}\right)$ and $e_{T}\left(T E_{0 s}\right)$ can be written down explicitly in terms of the universal bundles on the flag bundle $E_{0 s}=F l\left(m_{1}, \cdots, m_{k}, S\right)$ over $\operatorname{Gr}(r, n)$. Here $S$ is the universal bundle on the Grassmannian.

The push-forward by $p$ from $F l\left(m_{1}, \cdots, m_{k}, S\right)$ to $\operatorname{Gr}(r, n)$ is done by an analogue of family localization formula of Atiyah-Bott, which is given by a sum over the Weyl groups along the fiber which labels the fixed point sets.

In any case the final formula of degree $d$ is given by

$$
p_{*}\left[\frac{1}{e_{T}\left(E_{0 s} / Q_{d}\right)}\right]=(-1)^{(r-1) d} \sum_{\substack{\left(d_{1}, \ldots, d_{r}\right)=\\ d_{1}+\ldots+d_{r}=d}} \frac{\prod_{1 \leq i<j \leq r}\left(x_{i}-x_{j}+\left(d_{i}-d_{j}\right) \alpha\right)}{\prod_{1 \leq i<j \leq r}\left(x_{i}-x_{j}\right) \prod_{i=1}^{r} \prod_{l=1}^{d_{i}}\left(x_{i}+l \alpha\right)^{n}} .
$$

Here $x_{1}, \ldots x_{r}$ are the Chern roots of $S^{*}$. As a corollary of our approach, we have the following:

Corollary 7. The Hori-Vafa conjecture holds for Grassmannian manifolds.

This corollary was derived in [B-CF-K] by using the idea and method and also the key results in [L-L-L-Y]. The explicit form of Hori-Vafa conjecture for general flag manifolds and its justifications require further study in the future.

\section{MARIÑO-VAFA FORMULA}

To compute mirror formula for higher genus, we need to compute Hodge integrals, which are defined as follows. Let $\mathcal{M}_{g, h}$ be the moduli space of stable curves of genus $g$ with $h$ marked points. The Hodge bundle $\mathbb{E}$ is a rank $g$ vector bundle over $\mathcal{M}_{g, h}$ whose fiber over $\left[\left(C, x_{1}, \ldots, x_{h}\right)\right] \in \mathcal{M}_{g, h}$ is $H^{0}\left(C, \omega_{C}\right)$. The $\lambda$ classes are defined by

$$
\lambda_{j}=c_{j}(\mathbb{E}) \in H^{2 i}\left(\mathcal{M}_{g, h} ; \mathbb{Q}\right) .
$$

The cotangent line $T_{x_{i}}^{*} C$ of $C$ at the $i$-th marked point $x_{i}$ gives a line bundle $\mathbb{L}_{i}$ over $\mathcal{M}_{g, h}$. The $\psi$ classes are defined by

$$
\psi_{i}=c_{1}\left(\mathbb{L}_{i}\right) \in H^{2}\left(\mathcal{M}_{g, h} ; \mathbb{Q}\right) .
$$

Hodge integrals are intersection numbers of $\lambda$ classes and $\psi$ classes.

We next introduce a particular form of Hodge integrals. Given a partition

$$
\mu=\left(\mu_{1} \geq \cdots \geq \mu_{h}>0\right),
$$

define $\ell(\mu)=h$, and $|\mu|=\mu_{1}+\cdots+\mu_{h}$. Given a triple $(g, \mu, \tau)$, where $g$ is a nonnegative integer, $\mu$ is a partition, and $\tau \in \mathbb{Z}$, we define an one-partition Hodge integral as follows:

$$
\begin{aligned}
G_{g, \mu}(\tau)= & \frac{-\sqrt{-1}^{|\mu|+\ell(\mu)}}{|\operatorname{Aut}(\mu)|}(\tau(\tau+1))^{\ell(\mu)-1} \prod_{i=1}^{\ell(\mu)} \frac{\prod_{a=1}^{\mu_{i}-1}\left(\mu_{i} \tau+a\right)}{\left(\mu_{i}-1\right) !} \\
& \cdot \int_{\mathcal{M}_{g, \ell(\mu)}} \frac{\Lambda_{g}^{\vee}(1) \Lambda_{g}^{\vee}(-\tau-1) \Lambda_{g}^{\vee}(\tau)}{\prod_{i=1}^{\ell(\mu)}\left(1-\mu_{i} \psi_{i}\right)},
\end{aligned}
$$

where

$$
\Lambda_{g}^{\vee}(u)=u^{g}-\lambda_{1} u^{g-1}+\ldots+(-1)^{g} \lambda_{g} .
$$

The one-partition Hodge integral can be simplified in special cases: 
- $g=0: \Lambda_{0}^{\vee}(u)=1$.

$$
\begin{aligned}
& \int_{\mathcal{M}_{0, h}} \frac{1}{\prod_{i=1}^{h}\left(1-\mu_{i} \psi_{i}\right)} \\
= & \sum_{k_{1}+\cdots+k_{h}=h-3} \mu_{1}^{k_{1}} \cdots \mu_{h}^{k_{h}} \int_{\mathcal{M}_{0, h}} \psi_{1}^{k_{1}} \cdots \psi_{h}^{k_{h}} \\
= & \sum_{k_{1}+\cdots+k_{h}=h-3} \mu_{1}^{k_{1}} \cdots \mu_{h}^{k_{h}} \frac{h !}{k_{1} ! \cdots k_{h} !} \\
= & |\mu|^{h-3}
\end{aligned}
$$

- $\tau=0: G_{g, \mu}(0)=0$ if $\ell(\mu)>1$, and

$$
G_{g,(d)}(0)=\sqrt{-1}^{d+1} \int_{\mathcal{M}_{g, 1}} \frac{\lambda_{g}}{1-d \psi}=\sqrt{-1}^{d+1} d^{2 g-2} b_{g}
$$

where

$$
b_{g}= \begin{cases}1, & g=0 \\ \int_{\mathcal{M}_{g, 1}} \lambda_{g} \psi^{2 g-2}, & g>0\end{cases}
$$

To state Mariño-Vafa's conjecture on one-partition Hodge integrals, we introduce some generation functions.

We first define generating functions of one-partition Hodge integrals. Introduce variables $\lambda$ and $p=\left(p_{1}, p_{2}, \ldots\right)$. Given a partition $\mu$, let

$$
p_{\mu}=p_{\mu_{1}} \cdots p_{\mu_{\ell(\mu)}}
$$

Define generating functions

$$
\begin{aligned}
G_{\mu}(\lambda ; \tau) & =\sum_{g=0}^{\infty} \lambda^{2 g-2+\ell(\mu)} G_{g, \mu}(\tau) \\
G(\lambda ; \tau ; p) & =\sum_{\mu} G_{\mu}(\lambda ; \tau) p_{\mu}, \\
G^{\bullet}(\lambda ; \tau ; p) & =\exp (G(\lambda ; \tau ; p))=\sum_{\mu} G_{\mu}^{\bullet}(\lambda ; \tau) p_{\mu} .
\end{aligned}
$$

We next define generating functions of symmetric group representations. Let $\chi_{\mu}$ denote the character of the irreducible representation of symmetric group $S_{|\mu|}$ indexed by $\mu$ with $|\mu|=\sum_{j} \mu_{j}$, and let $C_{\mu}$ denote the conjugacy class of $S_{|\mu|}$ indexed by $\mu$. Introduce

$$
V_{\mu}(\lambda)=\prod_{1 \leq a<b \leq \ell(\mu)} \frac{\sin \left[\left(\mu_{a}-\mu_{b}+b-a\right) \lambda / 2\right]}{\sin [(b-a) \lambda / 2]} \cdot \frac{1}{\prod_{i=1}^{\ell(\nu)} \prod_{v=1}^{\mu_{i}} 2 \sin [(v-i+\ell(\mu)) \lambda / 2]},
$$

which has an interpretation in terms of quantum dimension in Chern-Simons knot theory. Define

$$
\begin{gathered}
R_{\mu}^{\bullet}(\lambda ; \tau)=\sum_{|\nu|=|\mu|} \frac{\chi_{\nu}\left(C_{\mu}\right)}{z_{\mu}} e^{\sqrt{-1}\left(\tau+\frac{1}{2}\right) \kappa_{\nu} \lambda / 2} V_{\nu}(\lambda), \\
R^{\bullet}(\lambda ; \tau ; p)=\sum_{\mu} R_{\mu}^{\bullet}(\lambda ; \tau) p_{\mu}
\end{gathered}
$$


where

$$
z_{\mu}=|\operatorname{Aut}(\mu)| \mu_{1} \cdots \mu_{\ell(\mu)}, \quad \kappa_{\mu}=|\mu|+\sum_{i}\left(\mu_{i}^{2}-2 i \mu_{i}\right)
$$

Define

$$
R(\lambda ; \tau ; p)=\log \left(R^{\bullet}(\lambda ; \tau ; p)\right) .
$$

Conjecture 8 (Mariño-Vafa $[\mathrm{M}-\mathrm{V}]$ ).

$$
G(\lambda ; \tau ; p)=R(\lambda ; \tau ; p) .
$$

Mariño-Vafa formula (2) provides a highly nontrivial link between geometry (Hodge integrals) and combinatorics (representations of symmetric groups). Note that for each fixed partition $\mu$, the Mariño-Vafa formula gives a closed and finite formula of $G_{\mu}(\lambda ; \tau)$, a generating function of all genera.

We now outline the proof of Mariño-Vafa formula due to C.-C. M Liu, K. Liu, and J. Zhou [L-L-Z1]. There is another approach due to A. Okounkov and R. Pandharipande $[\mathrm{O}-\mathrm{P}]$.

At $\tau=0$, both hand sides of the Mariño-Vafa formula can be greatly simplified:

$$
\begin{gathered}
G(\lambda ; 0 ; p)=-\sum_{d=1}^{\infty} \frac{\sqrt{-1}^{d+1} p_{d}}{\lambda d^{2}} \sum_{g=0}^{\infty} b_{g}(\lambda d)^{2 g} \\
R(\lambda ; 0 ; p)=-\sum_{d=1}^{\infty} \frac{-\sqrt{-1}^{d+1} p_{d}}{2 d \sin (\lambda d / 2)}
\end{gathered}
$$

They are equal by a previous result $[\mathrm{Fa}-\mathrm{P} 1]$ :

$$
\sum_{g=0}^{\infty} b_{g} t^{2 g}=\frac{t / 2}{\sin (t / 2)}
$$

Note that both hand sides of the Mariño-Vafa formula (2) are valid for $\tau \in \mathbb{C}$. It follows from the expression (1) that

$$
R_{\mu}^{\bullet}(\lambda ; \tau)=\sum_{|\nu|=|\mu|} R_{\nu}^{\bullet}(\lambda ; 0) z_{\nu} \Phi_{\nu \mu}^{\bullet}(\sqrt{-1} \lambda \tau)
$$

where

$$
\Phi_{\nu \mu}^{\bullet}(\lambda)=\sum_{\chi} \lambda^{-\chi+\ell(\nu)+\ell(\mu)} \frac{H_{\chi, \nu, \mu}^{\bullet}}{(-\chi+\ell(\nu)+\ell(\mu)) !}=\sum_{\eta} \frac{\chi_{\eta}\left(C_{\nu}\right)}{z_{\nu}} \frac{\chi_{\eta}\left(C_{\mu}\right)}{z_{\mu}} e^{\kappa_{\eta} \lambda / 2}
$$

is a generating function of disconnected double Hurwitz numbers $H_{\nu, \mu, \chi}^{\bullet}$. The convolution equation (6) is equivalent to the following cut-and-join equation:

$$
\frac{\partial R}{\partial \tau}=\frac{\sqrt{-1} \lambda}{2} \sum_{i, j=1}^{\infty}\left((i+j) p_{i} p_{j} \frac{\partial R}{\partial p_{i+j}}+i j p_{i+j}\left(\frac{\partial R}{\partial p_{i}} \frac{\partial R}{\partial p_{j}}+\frac{\partial^{2} R}{\partial p_{i} \partial p_{j}}\right)\right)
$$

In a symmetric group $S_{d}$, a transposition can $c u t$ an $(i+j)$-cycle into an $i$-cycle and an $j$-cycle:

$$
(s, t)\left(s, s_{2}, \cdots, s_{i}, t, t_{2}, \cdots t_{j}\right)=\left(s, s_{2}, \cdots, s_{i}\right)\left(t, t_{2}, \cdots t_{j}\right) .
$$

This corresponds to the cut operator

$$
(i+j) p_{i} p_{j} \frac{\partial}{\partial p_{i+j}} .
$$


A transposition can also join an $i$-cycle and a $j$-cycle to form an $(i+j)$-cycle:

$$
(s, t)\left(s, s_{2}, \cdots, s_{i}\right)\left(t, t_{2}, \cdots t_{j}\right)=\left(s, s_{2}, \cdots, s_{i}, t, t_{2}, \cdots t_{j}\right) .
$$

This corresponds to the join operator

$$
i j p_{i+j} \frac{\partial}{\partial p_{i}} \frac{\partial}{\partial p_{j}} .
$$

The Mariño-Vafa formula will follow from the initial values (3), (4), (5), the cut-and-join equation (7) of $R(\lambda ; \tau ; p)$, and the following cut-and-join equation of $G(\lambda ; \tau ; p)$ :

Theorem 9 (Liu-Liu-Zhou [L-L-Z1]).

$$
\frac{\partial G}{\partial \tau}=\frac{\sqrt{-1} \lambda}{2} \sum_{i, j=1}^{\infty}\left((i+j) p_{i} p_{j} \frac{\partial G}{\partial p_{i+j}}+i j p_{i+j}\left(\frac{\partial G}{\partial p_{i}} \frac{\partial G}{\partial p_{j}}+\frac{\partial^{2} G}{\partial p_{i} \partial p_{j}}\right)\right)
$$

The cut-and-join equation (8) is equivalent to the following convolution equation

$$
G_{\mu}^{\bullet}(\lambda ; \tau)=\sum_{|\nu|=|\mu|} G_{\nu}^{\bullet}(\lambda ; 0) z_{\nu} \Phi_{\nu, \mu}^{\bullet}(\sqrt{-1} \lambda \tau) .
$$

Theorem 9 is proved by applying functorial localization to the branch morphism

$$
\operatorname{Br}: \mathcal{M}_{g}\left(\mathbf{P}^{1}, \mu\right) \rightarrow \operatorname{Sym}^{r} \mathbf{P}^{1} \cong \mathbf{P}^{r},
$$

where $\mathcal{M}_{g}\left(\mathbf{P}^{1}, \mu\right)$ is the moduli space of relative stable maps from a genus $g$ curve to $\mathbf{P}^{1}$ with fixed ramification type $\mu=\left(\mu_{1}, \ldots, \mu_{h}\right)$ at $\infty$, and

$$
r=2 g-2+|\mu|+\ell(\mu)
$$

is the virtual dimension of $\mathcal{M}_{g}\left(\mathbf{P}^{1}, \mu\right)$. Note the $\mathbb{C}^{*}$-action on $\mathbf{P}^{1}$ induces $\mathbb{C}^{*}$-actions on the domain and the target of $\mathrm{Br}$, and $\mathrm{Br}$ is $\mathbb{C}^{*}$-equivariant. This is similar to the set-up of mirror principle, with a different linearized moduli.

We end this section with some applications of the Mariño-Vafa formula, following [L-L-Z2]. We have

$$
G_{g, \mu}(\tau)=\sum_{k=\ell(\mu)-1}^{2 g-2+|\mu|+\ell(\mu)} G_{g, \mu}^{k} \tau^{k}
$$

where

$$
\begin{aligned}
G_{g, \mu}^{2 g-2+|\mu|+\ell(\mu)} & =\frac{-\sqrt{-1}|\mu|+\ell(\mu)}{|\operatorname{Aut}(\mu)|} \frac{\mu_{i}^{\mu_{i}}}{\mu_{i} !} \int_{\mathcal{M}_{g, \ell(\mu)}} \frac{\Lambda_{g}^{\vee}(1)}{\prod_{i=1}^{\ell(\mu)}\left(1-\mu_{i} \psi_{i}\right)}, \\
G_{g, \mu}^{\ell(\mu)-1} & =\frac{-\sqrt{-1}|\mu|+\ell(\mu)}{|\operatorname{Aut}(\mu)|} \frac{\lambda_{g}}{\prod_{i=1}^{\ell(\mu)}\left(1-\mu_{i} \psi_{i}\right)} .
\end{aligned}
$$

The part corresponding to $G_{g, \mu}^{2 g-2+|\mu|+\ell(\mu)}$ in $R(\lambda ; \tau ; p)$ reduces to the Burnside formula of Hurwitz numbers $H_{g, \mu}$. We obtain the ELSV formula [ELSV]:

$$
\frac{1}{|\operatorname{Aut}(\mu)|} \frac{\mu_{i}^{\mu_{i}}}{\mu_{i} !} \int_{\mathcal{M}_{g, \ell(\mu)}} \frac{\Lambda_{g}^{\vee}(1)}{\prod_{i=1}^{\ell(\mu)}\left(1-\mu_{i} \psi_{i}\right)}=\frac{H_{g, \mu}}{(2 g-2+|\mu|+\ell(\mu)) !} .
$$


Extracting the part corresponding to $G_{g, \mu}^{\ell(\mu)-1}(\tau)$ from $R(\lambda ; \tau ; p)$, we obtain

$$
\sum_{g=0}^{\infty} \lambda^{2 g} \int_{\mathcal{M}_{g, n}} \frac{\lambda_{g}}{\prod_{i=1}^{n}\left(1-\mu_{i} \psi_{i}\right)}=|\mu|^{n-3} \frac{|\mu| \lambda / 2}{\sin (|\mu| \lambda / 2)} .
$$

The identity (11) is true for any partition of length $n$, so we may view it as an identity of polynomials in $\lambda, \mu_{1}, \ldots, \mu_{n}$. This gives us the values of all $\lambda_{g}$-integrals:

$$
\int_{\mathcal{M}_{g, n}} \psi_{1}^{k_{1}} \cdots \psi_{n}^{k_{n}} \lambda_{g}=\left(\begin{array}{c}
2 g+n-3 \\
k_{1}, \cdots, k_{n}
\end{array}\right) \frac{2^{2 g-1}-1}{2^{2 g-1}} \frac{\left|B_{2 g}\right|}{(2 g) !} .
$$

The identity (12) was first proved in [Fa-P2].

The following identities proved in [Fa-P1] are also consequences the Mariño-Vafa formula:

$$
\begin{aligned}
\int_{\mathcal{M}_{g}} \lambda_{g-2} \lambda_{g-1} \lambda_{g} & =\frac{1}{2(2 g-2) !} \frac{\left|B_{2 g-2}\right|}{2 g-2} \frac{\left|B_{2 g}\right|}{2 g} \\
\int_{\mathcal{M}_{g, 1}} \frac{\lambda_{g-1}}{1-\psi_{1}} & =b_{g} \sum_{i=1}^{2 g-1} \frac{1}{i}-\frac{1}{2} \sum_{\substack{g_{1}+g_{2}=g \\
g_{1}, g_{2}>0}} \frac{\left(2 g_{1}-1\right) !\left(2 g_{2}-1\right) !}{(2 g-1) !} b_{g_{1}} b_{g_{2}}
\end{aligned}
$$

\section{Mirror SYMMETRY}

In the previous sections we discussed the localization method to understand the counting function of Gromov-Witten invariants. These formulas are rather difficult to predict. They were motivated by important concepts of duality. A very important duality is called mirror symmetry. The counting function of GromovWitten invariants appears as instanton contribution to $\mathrm{II}_{A}$ theory of one Calabi-Yau manifold $M$. The ability of computing it came from the symmetry that the $\mathrm{II}_{A}$ theory of $M$ is isomorphic to $\mathrm{II}_{B}$ theory of another Calabi-Yau manifold $\widehat{M}$ which is "mirror" to $M$. The $\mathrm{II}_{B}$ theory can be computed by deformation of complex structure, which can in turn be computed by studying the periods of holomorphic differential.

However the construction of $\widehat{M}$ has not been explained in a fundamental way, except for some special cases. About eight years ago, Strominger, Zaslow and Yau, based on the newly developed brane theory, proposed a geometric construction of $\widehat{M}$. The program is still being pursued vigorously and it is closely related to the (more algebraic) homological mirror conjecture of Kontsevich and Fukaya.

We now explain the construction of SYZ and some of the important questions to be answered.

Motivated by understanding supersymmetric cycles in Calabi-Yau manifold, Becker-Becker-Strominger [B-B-S] considered the concept of Lagrangian subvarieties $V$ of a CY manifold $M$ so that the holomorphic three form, when restricted to the subvariety, is a (complex) constant (with norm one) multiple of the volume form of the subvariety. They consider a pair $(V, L)$ where $L$ is a $U(1)$ flat line bundle over $V$. These branes play an important role in understanding questions of duality as supersymmetric cycles are protected when coupling constants of the theory change.

Soon it was found that such subvarieties $V$ were studied by Harvey-Lawson earlier based on their interest on understanding examples of area minimizing subvarieties in Euclidean space. It was called special Lagrangian cycles by them. Later 
Mclean [Mc] proved that the local moduli of a special Lagrangian submanifold $V$ in a Calabi-Yau manifold is parametrized by harmonic one form on $V$. The space of harmonic one form also parametrize flat $U(1)$ bundles over $V$. Hence one can put an almost complex structure on the moduli space of the pair $(V, L)$. This was observed by Strominger-Yau-Zaslow [S-Y-Z] and was proposed there to study this moduli space as an interesting complex manifold. In particular, when the first Betti number of $V$ is equal to three, this complex manifold is three dimensional.

Based on the theory of branes, it was proposed by SYZ that if $V$ is a three dimensional torus, we can replace $V$ by its dual $V^{*}$, the moduli space of flat $U(1)$ line bundle over $V$, and obtain a new complex manifold $\widehat{M}$. In general, we shall need to make instanton correction to the complex structure on $\widehat{M}$. We proposed $\widehat{M}$ to be the mirror manifold of $M$.

The foliation defined by the special Lagrangian torus can be singular leaf. We expect that in the large radius limit, there is a map $f: M \longrightarrow S^{3}$ so that outside a trivalent graph $G \subset S^{3}$, the fibers are nonsingular special Langrangian torus. If these torus are linear we call the picture semi-classical. Leung-Yau-Zaslow [Le-Y-Z] has studied this mirror construction quite extensively. Many interesting predictions for mirror symmetry hold for this semi-classical setting.

The first explicit construction of Ricci flat metric for the semi-classical setting was due to Greene-Shapere-Vafa-Yau [G-S-V-Y]. It is an interesting question to find instanton correction to their metric to obtain the Ricci flat metric on the K-3 surface. M. Gross and P. Wilson [G-W] did study this problem based on perturbation of the semi-flat Ricci flat metric. Unfortunately we still have little information about the instantons which are holomorphic disks whose boundaries give non-trivial homology classes on the Lagrangian torus.

If we consider the domain which parametrizes the special Lagrangian torus in $M$, assuming we are in the semiflat situation, there is a Weil-Peterson metric on $S^{3} \backslash G$. The form of such metrics was worked out by N. Hitchin [Hi], that it is a Hessian metric defined on affine flat manifold. (This kind of metric was introduced by Cheng-Yau [C-Y] in 1980 as an analogue of Kähler metric for flat affine structure. Under some assumptions, Cheng-Yau also proved existence and uniqueness theorems for such metric.) Hence there is a flat affine structure on $S^{3} \backslash G$ and in order for the torus to be defined, the monodromic group is a subgroup of $S L(3, \mathbb{Z})$. It is believed that there is a well-defined volume form on $S^{3} \backslash G$ so that in a suitable flat coordinate, the metric has the form $\sum \frac{\partial^{2} u}{\partial x_{i} \partial x_{j}} d x_{i} d x_{j}$ and $\operatorname{det}\left(\frac{\partial^{2} u}{\partial x_{i} \partial x_{j}}\right)=1$. The existence of such metric on a tube domain is related to existence of Ricci flat Kähler flat metric if we look at the complexified coordinate $x_{j}+\sqrt{-1} y_{j}$. (This ansatz was first proposed by E. Calabi.) However, its existence and the behavior near the triple singular point of $\mathrm{G}$ is non-trivial. This was worked out recently by Lofton-Yau-Zaslow [Lo-Y-Z].

Potentially construction of SYZ geometry can be reduced to following data:

1. Construction of flat affine structure in $S^{3} \backslash G$ whose holonomy group is a subgroup of $S L(3, \mathbb{Z})$.

2. Construction of Cheng-Yau type hessian metric with a given flat volume form.

3. Construction of a map from $S^{3} \backslash G$ to the moduli space of flat torus that is compatible with the holonomy group mentioned above. 
Once this construction is carried out, one can construct the mirror manifold $\widehat{M}$ in the large radius limit. A very important verification of the SYZ construction of mirror conjecture is to understand the deformation of complex structure of $\widehat{M}$ and relate it to periods of holomorphic three form. It should reflect the counting of holomorphic curves of $M$.

Under reasonable topological assumption, M. Gross studied the SYZ construction for the quintic in $\mathbf{P}^{4}$ and compute the Hodge diagram of the mirror manifold. He concludes that the picture is consistent. W. Ruan [Ru] studied the Lagrangian fibration for Calabi-Yau manifolds that are constructed from toric manifolds.

The mirror correspondence is supposed to map even cohomology of $M$ to odd cohomology of $\widehat{M}$. We propose to construct this map in terms of the SYZ construction in the following manner.

For the map $f: M \longrightarrow S^{3}$ and its mirror $\widehat{f}: \widehat{M} \longrightarrow S^{3}$, we can form a nine dimensional variety by forming their fiber product $M \underset{S^{3}}{\times} \widehat{M} \longrightarrow S^{3}$. The general fiber of this map is given by $T^{3} \times\left(T^{3}\right)^{*}$ where it admits the standard Poincare (complex) line bundle $L$ so that $L$ restricted to $T^{3} \times\{l\}$ is given by $l$. We assume that $L$ can be extended to be a line bundle (or a sheaf) over $M \underset{S^{3}}{\times} \widehat{M}$.

Let $\Pi_{1}: M \underset{S^{3}}{\times} \widehat{M} \longrightarrow M$ and $\Pi_{2}: M \underset{S^{3}}{\times} \widehat{M} \longrightarrow M$. Then we can define the mirror map on cohomology level by taking any even degree cohomology class $\omega$ in $M$, and map it to $\left(\Pi_{2}\right)_{*}\left[\left(\Pi_{1}^{*} \omega\right) \exp \left(c_{1}(L)\right)\right]$ which gives odd cohomology in $\widehat{M}$.

This assertion should be easier to verify in the semi-classical picture when we have flat affine constructions. When one counts instantons corrections, one should be able to map quantum cohomology of $M$ to $H^{1}\left(T_{\widehat{M}}\right)$, the deformation space of complex structures of $\widehat{M}$.

In [Le-Y-Z], we discuss how to map special Lagrangian cycles in $\widehat{M}$ to stable holomorphic sheaves over $M$. It would be important to prove this picture rigorously (which in turn depends on a rigorous construction of $\widehat{M}$ ). Potential construction of special Lagrangian cycles in $\widehat{M}$ can give ways to construct holomorphic cycles in $M$. Therefore it becomes an important question to understand which odd dimensional cohomology classes in $\widehat{M}$ admit special Lagrangian cycles. The Hodge conjecture on $M$ may suggest that an integral multiple of each odd dimensional cohomology class in $\widehat{M}$ should be representable by special Lagrangian cycles.

There are several directions that we may want to generalize the above pictures.

1. When $M$ is Calabi-Yau, we can look for fiber space which are holomorphic. Hence $f: M \longrightarrow N$ is holomorphic, general fiber $T$ is polarized CalabiYau and the space $N$ is a Fano variety or a variety with negative Kodaira dimension. We can replace each fiber $T$ by its mirror manifold $\widehat{T}$. Hopefully one can complete the process to form a new compact Kähler manifold $\widehat{M}$ which is still Calabi-Yau. Obviously there are conditions one likes to impose in order for such assertion to hold.

The new manifold $\widehat{M}$ should reflect a great deal about the geometry of $M$. One can still define the transfer map from $M$ to $\widehat{M}$. Since everything is complex now, it maps even cohomology of $M$ to even cohomology of $\widehat{M}$.

At least when $T$ is complex torus, it may give isomorphism of derived 
category of $M$ to derived category of $\widehat{M}$, chow rings of $M$ to chow rings of $\widehat{M}$.

2. When $M$ is a more general Kähler-Einstein manifold, special Lagrangian does not make sense. However, we can replace it by Lagrangian cycles which is area minimizing among all Lagrangian cycles. Hence we are looking for Lagrangian cycles whose mean curvature one form is harmonic.

Many interesting questions in geometry can be motivated by such pictures.

\section{REFERENCES}

[B-B-S] K. Becker, M. Becker and A. Strominger, Fivebranes, membranes and non-perturbative string theory, Nuclear Phys. B456(1-2) (1995), 130-152.

[B-CF-K] A. Bertram, I. Ciocan-Fontanine and B. Kim, Two proofs of a conjecture of Hori and Vafa, math.AG/0304403.

[B-F] K. Behrend and B. Fantechi, The intrinsic normal cone, Invent. Math. 128 (1997), 45-88.

[Ca] E. Calabi, A construction of nonhomogeneous Einstein metrics, Proc. of Symp. in Pure Math., 27, 17-24, AMS, Providence, 1975.

[CdGP] P.Candelas, X.C. de la Ossa, P.S. Green, L. Parkes An exactly soluble superconformal theory from a mirror pair of Calabi-Yau manifolds, Phys. Lett. B 258 (1991), no. 1-2, $118-126$.

[Cox] D.A. Cox, The functor of a smooth toric variety, Tôhoku Math. J. (2)47 (1995), 251-262.

[C-V] S. Cecotti and C. Vafa, On the classification of $N=2$ supersymmetric theories, Commun. Math. 158 (1993), 569-644.

[C-Y] S.Y. Cheng and S.-T. Yau, The real Moge-Ampere equation and affine flat structures, 1980 Peking conference, Science Press, Beijing, 1982, 339-370.

[ELSV] T. Ekedahl, S. Lando, M. Shapiro, and A. Vainshtein, Hurwitz numbers and intersections on moduli spaces of curves, Invent. Math. 146(2) (2001), 297-327.

[Fa-P1] C. Faber and R. Pandharipande, Hodge integrals and Gromov-Witten theory, Invent. Math. 139(1) (2000), 173-199.

[Fa-P2] - Hodge integrals, partition matrices, and the $\lambda_{g}$ conjecture, Ann. of Math. (2) 157 (2003), no. 1, 97-124.

[F] K. Fukaya, Multivalued Morse theory, asymptotic analysis, and mirror symmetry, http://www.math.hyoton.ac.jp.

[Gi] A. Givental, Equivariant Gromov-Witten invariants, Internat. Math. Res. Notices 1996, no. 13, 613-663.

[G-S-V-Y] B. Greene, A. Shapere, C. Vafa and S.-T. Yau, Stringy cosmic strings and non-compact CY manifold, Nucl. Phys. B337 (1990), 1-3.

[Gr] M. Gross, Topological mirror symmetry, Invent. Math., 144 (2001), 75-137.

[Ge-P] E. Getzler and R. Pandharipande, Virasoro constraints and the Chern classes of the Hodge bundle, Nuclear Phys. B530 (1998), no. 3, 701-714.

[G-W] M. Gross and P.M.H. Wilson, Large complex structure limits of K3-surfaces, J. Differential Geom. 55 (2000), no. 3, 475-546.

[H-L] R. Harvey and H.B. Lawson, Jr, Calibrated geometries, Acta Math., 148 (1982), 47157.

[Hi] N.J. Hitchin, The moduli space of special Lagrangian submanifolds, Ann. Scuola Norm. Sup. Pisa Cl. Sci., 25 (1997), 503-515.

[H-I-V] K. Hori, A. Iqbal, and C. Vafa, D-branes and mirror symmetry, hep-th/0005247.

[HKKPTVVZ] K. Hori, S. Katz, A. Klemm, R. Pandharipande, R. Thomas, C. Vafa, R. Vakil, E. Zaslow, Mirror symmetry, Clay Math. Inst. Mono. 1, Amer. Math. Soc. 2003.

$[\mathrm{H}-\mathrm{V}] \quad$ H. Hori and C. Vafa, Mirror symmetry, hep-th/0002222.

[K-S] M. Kontsevich and Y. Soibelman, Homological mirror symmetry and Torus Fibration, in Symplectic geometry and mirror symmetry, World Scientific, 2001, 203-263.

[Le-Y-Z] N.C. Leung, S.-T. Yau and E. Zaslow, From special Lagrangian to Hermitian-YangMills via Fourier-Mukai Transform, math.DG/0005118. 
[Liu] K. Liu, Modular Invariance and Characteristic Numbers, Commun. Math. Phys. 174 (1995), 29-42.

[L-L-L-Y] B. Lian, C.-H. Liu, K. Liu, and S.-T. Yau, The $S^{1}$ fixed points in Quot-schemes and mirror principle computations, in Vector bundles and representations theory, S.D. Cutkosky, D. Edidin, Z. Qin, and Q. Zhang eds., Contemp. Math. 322, 165-194, Amer. Math. Soc., 2003.

[L-L-Y1] B. Lian, K. Liu, and S.-T. Yau, Mirror principle, I, Asian J. Math. 1 (1997), 729763; II, Asian J. Math. 3 (1999), 109-146; III, Asian J. Math. 3 (1999), 771-800; IV, math. AG/0007104.

[L-L-Y2] - A survey of mirror principle, math. AG/0010064.

[ChL-L-Y1] C.-H. Liu, K. Liu, and S.-T. Yau, On A-twisted moduli stack for curves from Witten's gauged linear sigma models, math.AG/0212316.

[ChL-L-Y2] — $S^{1}$-fixed-points in hyper-Quot-schemes and an exact mirror formula for flag manifolds from the extended mirror principle diagram, math.AG/0401367.

[L-L-Z1] C.-C. M. Liu, K. Liu, and J. Zhou, A proof of a conjecture of Mariño-Vafa on Hodge integrals, J. Differential Geom. 65 (2003), no. 2, 289-340.

[L-L-Z2] Mariño-Vafa formula and Hodge integral identities, math. AG/0308015.

[L-T] J. Li and G. Tian, Virtual moduli cycles and Gromov-Witten invariants of algebraic varieties, J. Amer. Math. Soc. 11 (1998), 119-174.

[Lo-Y-Z] J. Loftin, S.-T. Yau and E. Zaslow, Affine manifolds, SYZ geometry and the "Y" vertex,

[Mc] R.C. Mclean, Deformation of calibrated submanifolds, Com. Analy. Geom., 6 (1998), 705-774.

[M-V] M. Mariño and C. Vafa Framed knots at large $N$, in Orbifolds in mathematics and physics (Madison, WI, 2001), 185-204, Contemp. Math. 310, Amer. Math. Soc., Providence, RI, 2002.

[O-P] A. Okounkov, R. Pandharipande, Hodge integrals and invaraints of the unknots, math. AG/0307209.

$[\mathrm{Ru}] \quad$ W.-D. Ruan, Lagrangian torus fibrations of Calabi-Yau hypersurfaces in toric varieties and SYZ mirror symmetry conjecture, Mirror symmetry, IV (Montreal, QC, 2000), 33-55, AMS/IP Stud. Adv. Math., 33, Amer. Math. Soc., Providence, RI, 2002

[S-Y-Z] A. Strominger, S.-T. Yau and E. Zaslow, Mirror symmtry in T-duality, Nucl. Phys. B479 (1996), 243-259.

[Wi1] E. Witten, Phases of $N=2$ theories in two dimensions, Nucl. Phys. B403 (1993), 159-222.

[Wi2] - The Verlinde algebra and the cohomology of the Grassmannian, in Geometry, topology, and physics for Raoul Bott, S.-T. Yau ed., 357-422, International Press, 1994.

Department of Mathematics, Harvard University, Cambridge, MA02138, USA

E-mail address: ccliu@math.harvard.edu

Department of Mathematics, Harvard University, Cambridge, Ma02138, USA

E-mail address: chienliu@math.harvard.edu

Department of Mathematics, University of California, Los Angeles, Los Angeles, CA 90095-1555, USA, Center of Mathematical Sciences, Zhejiang University, Hangzhou, CHINA

E-mail address: liu@math.ucla.edu, liu@cms.zju.edu.cn

Department of Mathematics, Harvard University, Cambridge, MA 02138, USA

E-mail address: yau@math.harvard.edu 